\title{
Atypical Reflexive Gaze Patterns on Emotional Faces in Autism Spectrum Disorders
}

\author{
Dorit Kliemann, ${ }^{1,2,3}$ Isabel Dziobek, ${ }^{2}$ Alexander Hatri, ${ }^{1,2}$ Rosa Steimke, ${ }^{2,4}$ and Hauke R. Heekeren ${ }^{1,2,3}$ \\ ${ }^{1}$ Department of Educational Science and Psychology, and ²Cluster of Excellence, "Languages of Emotion," Freie Universität Berlin, 14195 Berlin, Germany, \\ and ${ }^{3}$ Max Planck Institute for Human Development, 14195 Berlin, Germany, and ${ }^{4}$ Department of Psychiatry and Psychotherapy, Charité University \\ Medicine, 10117 Berlin, Germany
}

Atypical scan paths on emotional faces and reduced eye contact represent a prominent feature of autism symptomatology, yet the reason for these abnormalities remains a puzzle. Do individuals with autism spectrum disorders (ASDs) fail to orient toward the eyes or do they actively avoid direct eye contact? Here, we used a new task to investigate reflexive eye movements on fearful, happy, and neutral faces. Participants (ASDs: 12; controls: 11) initially fixated either on the eyes or on the mouth. By analyzing the frequency of participants' eye movements away from the eyes and toward the eyes, respectively, we explored both avoidance and orientation reactions. The ASD group showed a reduced preference for the eyes relative to the control group, primarily characterized by more frequent eye movements away from the eyes. Eye-tracking data revealed a pronounced influence of active avoidance of direct eye contact on atypical gaze in ASDs. The combination of avoidance and reduced orientation into an individual index predicted emotional recognition performance. Crucially, this result provides evidence for a direct link between individual gaze patterns and associated social symptomatology. These findings thereby give important insights into the social pathology of ASD, with implications for future research and interventions.

\section{Introduction}

Recent reports from the social-cognitive neurosciences have emphasized the importance of the eyes in carrying crucial information about emotional states of others. In autism spectrum disorders (ASDs), atypical eye contact is not only a diagnostic criterion (American Psychiatric Association, 1994), but also one of the earliest pathological signs, even for high-functioning individuals. Abnormalities in processing of information from the eyes (Leekam et al., 1998; Baron-Cohen et al., 2001a; Spezio et al., 2007a) are characterized by specific scan paths on emotional faces: individuals with ASD spend less time on faces in general (Pelphrey et al., 2002) and in particular focus less on the eye region (Klin et al., 2002). Despite some recent studies on gaze behavior in ASD, it still remains unclear why individuals with ASD show these abnormalities in face processing. A longstanding view suggests a general lack of social attention and specifically less attention toward the eyes as the main reason for the observed scan path on emotional faces in ASD. Some studies even found that individuals with ASD tend to focus more on the mouth region (Klin et al., 2002; Pelphrey et al., 2002). These findings may be taken as support for the missing orientation toward the eyes (Schultz, 2005). Thus, a failure to detect social saliency leads to a reduced orientation toward important sources

Received Feb. 8, 2010; revised June 24, 2010; accepted July 19, 2010.

This work was supported by the German Federal Ministry of Education and Research and the Max Planck Society. We thank Jennifer Kirchner for assistance in establishing diagnoses and Hannah Bruehl for valuable comments on the manuscript.

Correspondence should be addressed to Dorit Kliemann, Cluster of Excellence, "Languages of Emotion," Freie Universität Berlin, Habelschwerdter Allee 45, 14195 Berlin, Germany. E-mail: dorit.kliemann@fu-berlin.de.

DOI:10.1523/JNEUROSCI.0688-10.2010

Copyright $\odot 2010$ the authors $\quad 0270-6474 / 10 / 3012281-07 \$ 15.00 / 0$ of social information such as the eyes (Schultz, 2005; Neumann et al., 2006). Another emerging view, however, highlights the potential aversiveness of direct eye contact in ASD, resulting in an active avoidance reaction (Dalton et al., 2005; Kylliainen and Hietanen, 2006). Support for an aversion explanation of atypical gaze comes from studies showing increased skin conductance responses in children with autism when confronted with direct eye contact compared with averted gaze (for recent reviews on gaze and autism, see Nation and Penny, 2008) (Senju and Johnson, 2009). Additionally, another recent study (Joseph et al., 2008) showed that face-encoding skills of children and adolescents with ASD were mediated by emotional arousal in response to direct gaze.

Notably, those two processes do not have to be mutually exclusive, and the interplay of the two components may in fact account for the observed scan paths (Spezio et al., 2007b). Findings to date provide some evidence for each of these explanations; no study so far, however, addressed both processes specifically within the same research design. Here, we sought to further investigate the influence of (1) reduced social orientation to and (2) the active avoidance of the eyes on atypical gaze in ASD by analyzing participants' eye movements while they performed a new behavioral facial emotion classification task (Gamer and Büchel, 2009). We varied the initial fixation position on the face; thus, participants started processing a face either at the eyes or at the mouth and decided whether the face showed a happy, fearful, or neutral expression. Thereby, the task allows investigating both avoidance- and orientation-guided reflexive gaze behaviors, triggered by focusing the eyes or the mouth, respectively.

\section{Materials and Methods}

Nineteen neurotypical controls (NTs) (14 male; age range, 21-42 years; mean age, 30.37 years) with no known psychiatric or neurological disor- 
der and 17 participants with ASD (12 male; age range, 22-48 years; mean age, 32.71 years) participated in the current study. Participants in the NT group were recruited through databases of the Max Planck Institute for Human Development (Berlin, Germany). Participants with ASD were recruited through psychiatrists who specialized in autism in the Berlin area and the outpatient clinic for autism in adulthood of the Charite University Medicine, Berlin, Germany. Diagnoses were made according to Diagnostic and Statistical Manual of Mental Disorders, Fourth Edition, criteria for Asperger syndrome and autism without mental retardation (American Psychiatric Association, 1994) using a videotaped semistructured interview, which included the assessment of childhood information. In 14 participants with available parental information, diagnoses were additionally confirmed with the Autism Diagnostic InterviewRevised (ADI-R) (Lord et al., 1994; Bölte and Poustka, 2001) and the Asperger Syndrome (and High-Functioning Autism) Diagnostical Interview (ASDI) (Gillberg et al., 2001). Restricting the eye movement data analysis to only the subsample of ASD participants with ADI-R data revealed the same pattern of results compared with the complete sample though very minor differences in $p$ values for some analyses (most likely because of the reduced sample size). Groups were matched with respect to gender, age, and verbal intelligence quotient (IQ) (see Results). All participants had normal or corrected-to-normal vision, were native German speakers, received payment, and gave written informed consent in accordance with the requirements of the ethics committee of the Max Planck Institute for Human Development.

Visual stimuli were shown using Presentation (Version 12.4., Neurobehavioral Systems), and gaze behavior was measured via an integrated T120 $60 \mathrm{HZ}$ eye tracker (Tobii Systems). The eye-tracking device was built into the screen and did not require fixing participants' heads. The device tracks both eyes separately using corneal reflection.

One hundred twenty faces from a standardized dataset (Lundqvist et al., 1998) were chosen for the emotion classification task, based on a recent validation study (Goeleven et al., 2008). Twenty women and 20 men each displayed happy, fearful, and neutral expressions in a randomized order. Each image was rotated to ensure that, when displayed, the eyes were at the same vertical height. Additionally, an elliptic mask was applied resulting in images containing just the face (Fig. 1). All images were converted to grayscale, and the cumulative brightness was normalized across images.

In each trial, a fixation cross was presented initially ( $2 \mathrm{~s})$, followed by the presentation of a face ( $150 \mathrm{~ms})$ (Fig. 1). After a blank gray screen $(2 \mathrm{~s})$, participants were asked to indicate the emotional expression via button press on a standard keyboard. The emotional classification experiment followed a $2 \times 3$ within-subjects design with the factors "initial fixation" (eyes, mouth) and "emotion" (happy, fearful, neutral). To investigate the effect of initial fixation on gaze behavior, half of the faces within each emotion category were shifted either downward (Fig. 1, trial A) or upward (Fig. 1, trial B), so that the eyes or the mouth appeared at the location of the formerly presented fixation cross. Thereby, the task allowed the investigation of reflexive gaze behavior as a response to direct eye contact as well as eye movements, when initially fixating the mouth. Eye movements analysis. Eye movement behavior and pupil size were recorded at a rate of 60 data points per second $(60 \mathrm{~Hz})$, averaged over both eyes. Resulting time series were split into trials starting $1 \mathrm{~s}$ before face presentation up to $2 \mathrm{~s}$ thereafter. The gaze point coordinates were then convoluted with a Gaussian function (SD, $15 \mathrm{~ms}$ ), and episodes were determined in which the first derivative of the resulting time series did not exceed 10 pixels ( 0.3 degree or $18 \%$ s angular speed). Only those trials were included in which participants fixated within 50 pixels $\left(1.5^{\circ}\right)$ of the fixation cross before the onset of the face and that did not contain consecutive missing data points for $>100 \mathrm{~ms}$. Missing data points are typically caused by blinks, head motion, poor calibration, and, likely, by fast eye motion. To compare groups and conditions, the remaining valid trials were then categorized as trials containing a fixation change (upward or downward) or as trials not containing a fixation change within $1 \mathrm{~s}$ of face presentation. A fixation change was defined as occurring at least 50 pixels $\left(1.5^{\circ}\right)$ away from the original position of the fixation cross in vertical direction. We then calculated the proportion of fixation changes downward (when the eyes were presented at the location of the

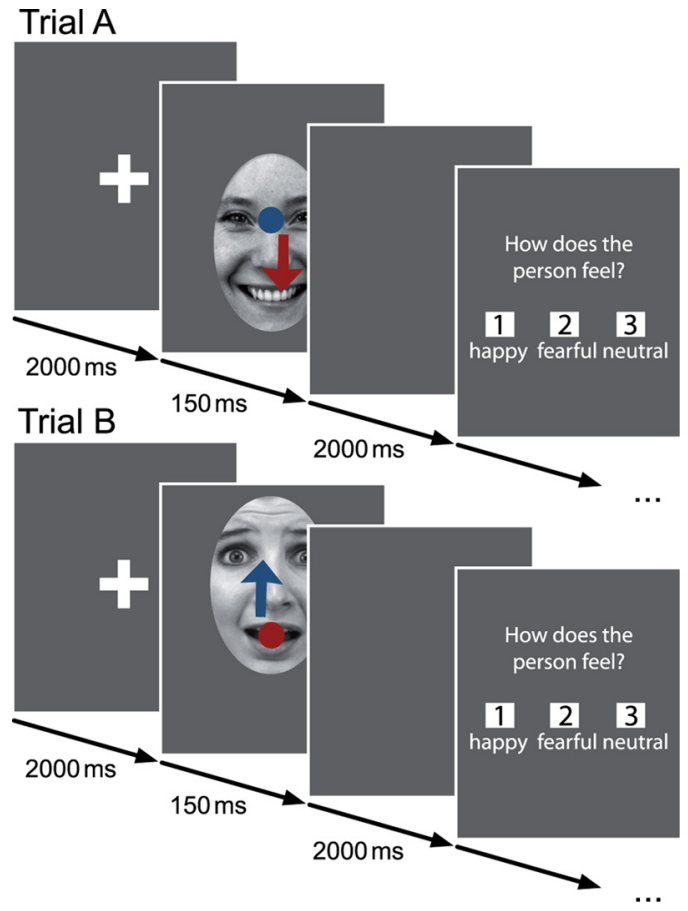

Figure 1. Emotion classification task. Each trial started with the presentation of a fixation cross ( $2 \mathrm{~s}$ ), followed by an emotional face ( $150 \mathrm{~ms})$. After a blank screen for $2 \mathrm{~s}$, subjects were asked to indicate the emotion displayed. Faces were shifted vertically on the screen, so that participants started gaze either on the eyes (trial A) or on the mouth (trial B) of the presented emotional face. Hypothesized gaze behavior of ASD group in red and of NT group in blue.

fixation cross) and upward (when the mouth was presented at the location of the fixation cross) as reflexive gaze responses triggered by the eyes or the mouth. Participants who had $<20 \%$ valid trials for each of the six conditions were excluded from further analysis. Because of exclusion criteria (e.g., technical failure or participants' movements), the presented results are based on 12 individuals with ASD and 11 controls. Over all six conditions, included participants had, on average, $62.1 \%$ valid trials.

Additionally, we calculated an individual eye preference index for difference between the proportion of trials with a fixation change upward from the mouth and the proportion of trials with a fixation change downward from the eyes (Gamer and Büchel, 2009), as well as an individual eye preference control index (number of trials with a fixation change upward from the mouth divided by the number of trials with a fixation change upward from the mouth plus the number of trials with a fixation change downward from the eyes) separately for each emotion and on average over all emotions. Both indices indicate how often participants gazed away from the eyes compared with the mouth. The control index, however, did not take into account the number of trials with no fixation change and therefore validates the eye preference index by controlling for the effect of missing trials. Indeed, we did not expect that trials with a fixation change downward were missed with a different probability than trials with a fixation change upward, but it was possible that trials with fixation changes were missed with a different probability than trials with no fixation changes. If this would have been the case, we expected that those indices would differ considerably. Note that we cannot make any conclusions about the final target of the observed eye movements but only about the directionality attributable to methodological limitations in recording frequency.

Statistical analysis. Since we were primarily interested in the main effects of the two factors initial fixation and emotion, as well as their interaction within and between groups, any data collected during the task were first analyzed applying a $2 \times 3$ [initial fixation (eyes vs mouth) $\times$ emotion (happy vs fear vs neutral)] repeated-measures ANOVA with between-subject factor group (ASD vs NT). Additionally, post hoc within-group ANOVAs, independent-samples $t$ tests, as well as pairedsamples $t$ tests were conducted. All statistics used a significance level of 
Table 1. Demographic variables and diagnostic scores

\begin{tabular}{lccl}
\hline & ASD & NT & $p$ \\
\hline $\begin{array}{l}\text { Complete sample } \\
\text { Gender (male/female) }\end{array}$ & $12 / 5$ & $14 / 5$ & \\
Age (yr) & $32.7 \pm 8.2$ & $30.4 \pm 5.9$ & 0.84 \\
MWT-IQ & $104.5 \pm 15.6$ & $110.4 \pm 12.9$ & 0.33 \\
Valid eye movement data sample & & & \\
Gender (male/female) & $8 / 4$ & $8 / 3$ & 0.75 \\
Age (yr) & $35.4 \pm 8.1$ & $27.1 \pm 2.6$ & 0.004 \\
MWT-IQ & $102.6 \pm 14.3$ & $105.9 \pm 10.6$ & 0.53 \\
\hline
\end{tabular}

Demographic variables and diagnostic scores for (1) the initial sample [ASD $(n=17)$ and NT participants $(n=19)]$ (upper) and (2) the subsample with valid eye-tracking data (ASD: $n=12 ; \mathrm{NT}: n=11$ ) (lower). $p$ values reflectlevels of significance from independent samples $t$ test and $\chi^{2}$ as appropriate. Values are given as mean $\pm S D$. MWT-IQ, Multiple choice vocabulary IQ test.

Table 2. Latency of fixation changes

\begin{tabular}{lll}
\hline & \multicolumn{2}{l}{ Mean (SD) (ms) } \\
\cline { 2 - 3 } & ASD & NT \\
\hline Eye & $449(199)$ & $508(185)$ \\
Happy & $438(203)$ & $437(124)$ \\
Fearful & $437(213)$ & $497(204)$ \\
Neutral & $423(166)$ & $566(210)$ \\
Mouth & $437(114)$ & $404(93)$ \\
Happy & $428(179)$ & $438(131)$ \\
Fearful & $508(167)$ & $402(91)$ \\
Neutral & $381(79)$ & $396(142)$ \\
\hline
\end{tabular}

$p<0.05$, unless otherwise specified. Data were analyzed using SPSS (version 17.0 for Mac; SPSS Inc.).

\section{Results}

\section{Demographics}

Groups were matched with respect to gender and age (Table 1). To assure comparable verbal IQ levels between groups, we tested participants with a vocabulary IQ test [multiple choice vocabulary test (MWT)] (Lehrl et al., 1995) (Tables 1, 2). Additionally, we used the Autism Spectrum Quotient (AQ) (Baron-Cohen et al., 2001b) in both groups to control for clinically significant levels of autistic traits in the NT group. None of the controls scored above the cutoff score of 32; in fact, the highest score was 24 , indicating a very low level of autistic traits in the NT group (ASD group: mean, 38.3; SD, 8.4; NT group: mean, 13.2; SD, 5.1).

We had valid eye movement data for a subsample of 12 individuals with ASD and 11 control participants (for subsample demographic information, see Table 1). Importantly, the subsample did not differ with respect to gender and verbal IQ, but ASD participants were significantly older than NT participants (Table 1). The factor age did, however, not influence eye movements $\left(F_{(1,20)}=0.37, p=0.57\right)$ and was therefore excluded from the model.

\section{Behavioral results}

\section{Reaction times}

We additionally analyzed participants' reaction times during the emotion classification task by condition with respect to the onset of the face stimuli. Individuals with ASD were generally slower in responding than NTs (ASD: $568 \mathrm{~ms}$; NT: $432 \mathrm{~ms} ; t_{(34)}=2.23 ; p=$ $0.03)$. The above-described $2 \times 3$ ANOVA with between-subject factor group yielded a significant three-way interaction $\left(F_{(2,68)}=\right.$ 5.33; $p=0.007$; partial $\left.\eta^{2}=0.14\right)$, as well as a two-way interaction between emotion and initial fixation $\left(F_{(2,68)}=6.86\right.$; $p=0.002$; partial $\left.\eta^{2}=0.17\right)$. Reaction times of the ASD group were influenced by an interaction of emotion and initial fixation $\left(F_{(2,32)}=7.17 ; p=\right.$ 0.003 ; partial $\left.\eta^{2}=0.31\right)$, whereas the interaction did not reach sig- nificance for the NT group ( $p=0.12$ ). Both NT and ASD groups were slower in responding to fearful faces when they started their gaze on the mouth compared with the eyes (ASD: fear-eyes: 561.45; fear-mouth: $645.88 ; t_{(16)}=-2.19 ; p=0.04$; NT: fear-eyes: 405.5 ; fear-mouth: $\left.439.95 ; t_{(18)}=-2.03 ; p=0.057\right)$. For neutral faces, however, individuals with ASD were significantly slower in responding to faces when the eyes were presented initially (neutral-eyes: 630.14; neutral-mouth: $503.1 ; t_{(16)}=3.05 ; p=0.008$ ). Notably, individuals with ASD responded marginally faster to happy faces compared with neutral faces regardless of initial fixation (happy: 536.17; fearful: 603.67; $\left.t_{(16)}=-2.02 ; p=0.06\right)$.

\section{Emotion classification performance}

For the emotion classification task, individuals in the NT group showed on average a higher correct classification percentage than individuals in the ASD group (NT: 95.46; ASD: $91.14 ; t_{(34)}=$ $-2.13 ; p=0.04)$. Performance over both groups was affected by a significant main effect of emotion $\left(F_{(2,68)}=3.54 ; p=0.035\right.$; partial $\left.\eta^{2}=0.09\right)$ and a significant interaction of initial fixation and emotion $\left(F_{(2,68)}=18.9 ; p=<0.001\right.$; partial $\left.\eta^{2}=0.36\right)$. The factor group marginally interacted with initial fixation $\left(F_{(2,68)}=\right.$ 3.6; $p=0.07$; partial $\eta^{2}=0.09$ ). Especially in conditions when the mouth was fixated initially, the ASD group showed fewer correct classifications than NTs (across emotions: $p=0.014$; happy-mouth: $p=0.055$; fear-mouth: $p=0.03$ ).

Both groups were separately influenced by an interaction of emotion and initial fixation $\left(\mathrm{NT}: F_{(2,36)}=6.89 ; p=0.003\right.$; partial $\eta^{2}=0.28$; ASD: $F_{(2,32)}=12.14 ; p<0.001$; partial $\left.\eta^{2}=0.43\right)$, whereas only individuals in the NT group showed a marginally significant main effect of the factor emotion $\left(F_{(2,36)}=3.03 ; p=\right.$ 0.061 ; partial $\left.\eta^{2}=0.14\right)$. For happy compared with neutral faces, controls classified more emotional expressions correctly (happy: 96.58; neutral: 93.36; $t_{(18)}=2.12 ; p=0.048$ ). Larger hit rates within the NT group were also observed when the eyes were presented at the fixation cross compared with the mouth across emotions (eyes: 93.46; mouth: 96.49; $t_{(18)}=-2.53$; $p=0.02$ ). Overall, individuals with ASD were able to discriminate a higher percentage of happy faces compared with fearful faces (happy: 94.37; fearful: $\left.90.39 ; t_{(17)}=1.86 ; p=0.08\right)$. For fearful faces, both participants in the ASD and NT groups could discriminate a higher percentage correctly when they initially fixated the eye region (ASD: $p=0.003$; NT: $p=0.076$ ), whereas for neutral faces the hit rates were larger when participants initially fixated the mouth (ASD: $p=0.05$; NT: $p=0.02$ ).

\section{Eye Movements results}

Analysis of eye movements revealed a three-way interaction of initial fixation, emotion, and group $\left(F_{(2,42)}=2.89 ; p=0.067\right.$; partial $\left.\eta^{2}=0.12\right)$, which shortly fell from significance. The factor initial fixation additionally interacted with the factors group $\left(F_{(1,21)}=8.03 ; p=0.01 ;\right.$ partial $\left.\eta^{2}=0.28\right)$ and emotion $\left(F_{(2,42)}=\right.$ $6.44 ; p=0.004$, partial $\eta^{2}=0.24$ ). Averaged over emotions (ASD: $70.09 \%$; NT: $30.85 \%$; $\left.t_{(21)}=2.37 ; p=0.005\right)$, as well as for all emotions separately (all $p<0.05$ ), individuals with ASD gazed significantly more often downward than controls when initially fixating the eyes (Fig. $2 A, C$ ). There was no significant difference in the number of fixation changes upward from the mouth to the eyes between groups (all $p>0.1$ ).

Within the NT group, eye movements were mediated by a significant main effect of initial fixation $\left(F_{(1,10)}=5.06 ; p=0.048\right.$; partial $\left.\eta^{2}=0.34\right)$ and a significant two-way interaction of initial fixation and emotion $\left(F_{(2,20)}=7.82 ; p=0.003\right.$; partial $\eta^{2}=$ $0.44)$. The ASD group showed only a trend for this interaction 


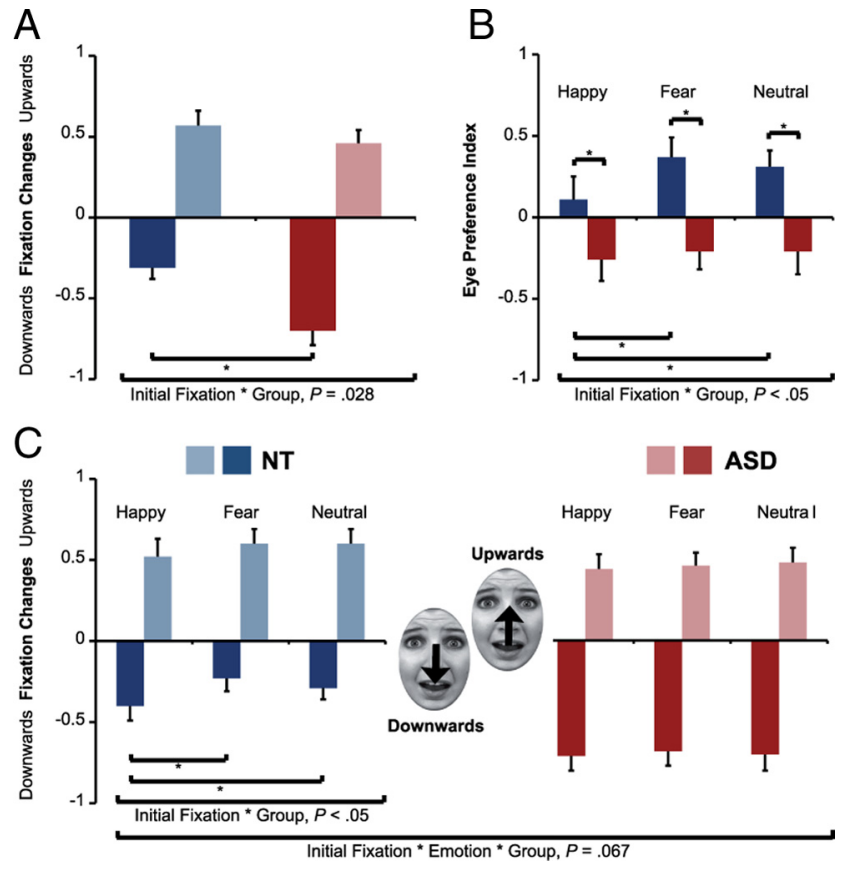

Figure 2. Fixation change patterns. $\boldsymbol{A}$, Function of initial fixation (without emotion). The ASD group showed more fixation changes downward away from the eyes than the NT group. $\boldsymbol{B}$, Eye preference index. Generally, the NT group showed an increased preference for the eyes compared with ASD. For the NT group, the preference was stronger for fearful and neutral faces compared with happy faces. There was no effect of emotion in the ASD group. C, Interaction of initial fixation and emotion. Within the NT group, the proportion of fixation changes downward away from the eye region (solid) was mediated by the displayed emotional expression, whereas this effect was absent within the ASD group.

$(p=0.1)$. Generally, controls oriented their gaze to a greater proportion upward toward the eyes (when initially fixating the mouth) than downward away from the eyes (when initially fixating the eyes) (upward: $57.83 \%$; downward: $30.85 \%$; $t_{(10)}=$ $-2.25 ; p=0.048)$. The size of the effect was further modulated by the emotional expression, most prominently for fearful faces (upward: $60.74 \%$; downward: $\left.23.48 \% ; t_{(10)}=-2.97 ; p=0.014\right)$ and neutral faces (upward: 60.81\%; downward: $29.05 \% ; t_{(10)}=$ $-3.02 ; p=0.013)$, but not for happy faces $(p=0.43)$. In contrast to the controls, the ASD group showed a trend toward a greater proportion of fixation changes downward away from the eyes than upward from the mouth toward the eyes (upward: $46.78 \%$; downward: $\left.70.09 \% ; t_{(11)}=-1.8 ; p=0.1\right)$, regardless of the emotion displayed.

To further investigate eye movements with respect to a possible interaction of the two initial fixation positions, we computed an index for eye preference for each participant (the difference between the proportion of trials with a fixation change upward from the mouth and downward from the eyes). Overall, the NT group's eye movements indicated a greater preference for the eyes than the ASD group's eye movements (averaged over emotions: $p=0.01$; fearful: $p=0.002$; neutral: $p=0.008$; happy: $p=0.068$ ). Again, only the NT group's gaze patterns were affected by the emotional expression: the index was significantly smaller for happy than for fearful faces (happy: 11.89 ; fear: $37.26 ; t_{(10)}=$ $-3.17 ; p=0.01$ ) and neutral faces (happy: 11.89; neutral: 31.76 ; $t_{(10)}=-2.74 ; p=0.01$ ) (Fig. $2 B$ ).

The number of valid trials was influenced by interactions of group with initial fixation $\left(F_{(1,21)}=10.57 ; p=0.004\right.$; partial $\eta^{2}=$ $0.33)$ and marginally by emotion $\left(F_{(2,42)}=3.15 ; p=0.053\right.$; partial $\left.\eta^{2}=0.13\right)$. The ASD group had more valid trials when initially fixating the eyes across emotions $(p=0.02)$, especially for happy faces $(p=0.009)$. To check whether the observed differences in number of valid trials may account for the effects on gaze behavior between groups described earlier, we calculated an additional individual index (eye preference control index), which accounts for differences in number of valid trials between trials with and without fixation changes (see Eye movements analysis). The individual values of the original eye preference index and the control index were highly correlated (Pearson's $r=$ 0.96; $p<0.001,2$-tailed), thereby reflecting the same pattern of effects. Consequently, the reported between-group differences in fixation changes were not a result of differences in the number of valid trials but represent actual differences in gaze patterns on emotional faces between the ASD and NT groups.

To further approximate the differences between avoidance and orienting response, we further analyzed the latency (time between occurrence of facial stimuli and offset of first fixation change) of participants' fixation changes. Although not reaching statistical significance, the ASD group showed a tendency toward faster eye movements away from the eyes than the NT group (averaged over emotions: ASD mean, 449 ms; NT mean, 508 ms) (Table 2), whereas the ASD group gazed more slowly upward toward the eyes (when starting fixation on the mouth) compared with the NT group (averaged over emotions: ASD mean, $437 \mathrm{~ms}$; NT mean, $404 \mathrm{~ms}$ ). For both directions, the effects were most pronounced for fearful faces, but almost absent for happy faces (away from eyes, fearful: ASD: 437 ms; NT: 497 ms; happy: ASD: $438 \mathrm{~ms}$, NT: $437 \mathrm{~ms}$; toward eyes, fearful: ASD: $508 \mathrm{~ms}$, NT: 402 ms; happy: ASD: $428 \mathrm{~ms}$, NT: $438 \mathrm{~ms}$ ), indicating a differential response to facial emotion. In line with previous reports, fearful faces, and especially wide-open eyes, seem more arousing than happy faces, which do not indicate a potential threat in the environment (Morris et al., 1996). Importantly, individuals with autism gazed away from the eyes faster than they gazed away from the mouth for fearful faces (eyes: mean, $437 \mathrm{~ms}$; mouth: mean, $508 \mathrm{~ms}$ ), while controls showed the opposite pattern (eyes: mean, 497 ms; mouth: mean, $402 \mathrm{~ms}$ ). In sum, those data indicate that avoidance of the eyes is indeed more pronounced in individuals with ASD because they gaze away from the eyes faster than the NT group and do so relatively faster than gazing away from the mouth toward the eyes, especially for fearful faces.

Interestingly, and in line with an interpretation of aversion, the more often ASD participants gazed away from the eye region, the faster these eye movements occurred (averaged over emotions: Pearson's $r=-0.93, p<0.001,2$-tailed). Contrarily, there was no such correlation between latency and fixation changes upward from the mouth toward the eyes in ASD (correlations differed significantly; Fisher's $z$ to $p$ transformation: $z=2.89, p=$ 0.004). The NT group, in turn, showed no modulation of number of fixation changes by latency at all.

The individual eye preference index was furthermore associated with autistic symptomatology as well as emotion recognition performance. For the ASD group, the eye preference index correlated positively with individual overall performance in the emotion classification task (Pearson's $r=0.66 ; p=0.019$, 2-tailed). This effect was absent for the NT group. To further assess whether general illness severity rather than socioemotional impairments account for the observed ASD-specific gaze patterns, we investigated the influence of the ADI-R total score (as a measure of illness severity) versus the influence of emotion recognition performance in a stepwise regression analysis on the eye preference index as the dependent variable. The emotion recognition performance was a significant predictor of eye movement 
patterns $\left(R^{2}\right.$ change $=0.74 ; F$ change $_{(1,7)}=20.2 ; \beta=0.4, p=$ $0.003)$, whereas the ADI-R total score was excluded from the model. Additionally, the eye preference index correlated negatively with the ADI-R social score (Pearson's $r=-0.7 ; p=0.033$, 2-tailed), whereas there was no correlation for the ADI-R communication score $(p>0.13)$ and ADI-R behavior score $(p>$ $0.56)$. Thus, the greater the preference for the eye region in the ASD group, the less pronounced the social symptomatology, indicating a link between gaze pattern and social impairments but not general illness severity in autism. In addition, there was no relationship of verbal IQ and group specific gaze patterns for both groups (ASD, $p=0.12$; NT, $p=0.89$ ), nor for the AQ scores (ASD, $p=0.14$, NT: $p=0.56$ ). Taking both the performance and diagnostic correlative relationships on the gaze patterns in ASD together, the greater the preference for the eye region in the ASD group, the higher the percentage of correct emotion classification and the less severe the social symptomatology.

Analysis of additional eye movement parameters revealed that there were no global group differences in the average $y$-axis offset during presentation of the fixation cross ( $250 \mathrm{~ms}$ before up to 50 ms after face presentation; ASD: 1.1 pixels; $\mathrm{NT}: 4.6$ pixels; $t_{(21)}=$ $-1.55 ; p=0.14$ ), the average duration (ASD: $908 \mathrm{~ms}$; NT: 1011 $\mathrm{ms} ; t_{(16)}=-1.27 ; p=0.22$ ), distance (ASD: 93.9 pixel; NT: 89.44 pixel; $t_{(21)}=3.95 ; p=0.7$ ), and latency of fixation changes (ASD: $428 \mathrm{~ms}$; NT: $\left.459 \mathrm{~ms} ; t_{(21)}=-7.78 ; p=0.44\right)$. Additionally, the number of trials with a fixation change downward from the mouth or upward from the eyes did not differ significantly from 0 . Those very few trials $(<0.5 \%$ of the trials per participant on average) were excluded from any further analysis. There were no group differences in $x$-axis offsets of fixation changes from the mouth upward toward the left or right half of the screen (i.e., toward the left or right eye) (ASD: -3.5 pixels; NT: -0.9 pixel; $\left.t_{(21)}=-0.32 ; p=0.75\right)$.

\section{Discussion}

The main goal of this study was to investigate the influence of diminished social orientation and active avoidance on the reduced focus to the eyes in ASD. The ASD group showed an overall reduced preference for the eye region compared with the NT group, which was specifically the result of an increase of fixation changes away from the eyes. Further analyses revealed groupspecific directional effects when combining both components: the NT group shifted their gaze significantly more often toward the eyes than away from the eyes, replicating previous results in typically developed individuals (Gamer and Büchel, 2009). Conversely, individuals with ASD showed more fixation changes away from the eyes than toward the eyes (Fig. 2).

These results can be seen as support for the hypothesis of increased avoidance of eye contact in ASD. We are aware, however, that both avoidance and orienting responses likely coexist at any time, yet to varying degrees. For example, it is likely that an initial reflexive orientation is present in ASD (as indicated by the number of fixation changes toward the eyes), but that it is then attenuated by an avoidance of eye contact (e.g., fixations do not reach or the eyes). The combination of both forces might furthermore interact differentially in both groups, as assessed with the eye preference index. A clear disentangling of both processes can therefore not be performed based on the number of fixation changes alone. In fact, gaze away from the eyes might indicate avoidance, but it might also indicate an increased interest in the mouth, because, for example, it might be more informative.

We thus performed analyses on additional eye movement parameters, which further support the pronounced influence of active avoidance of eye contact in ASD. Though only on a descriptive level, the ASD group showed a tendency to gaze away from the eyes faster than the NT group and, importantly, did so relatively faster than gazing away from the mouth toward the eyes. This effect was most pronounced for fearful faces, indicating differential responses to facial emotion. In line with previous reports, fearful faces_-and especially eyes_are more arousing than happy faces, which do not indicate a potential threat in the environment (Morris et al., 1996). Statistically reliable correlations underline these patterns, since the more often ASD participants gazed away from the eyes, the faster these eye movements occurred. Contrarily, there was no such correlation between latency and gaze away from the mouth in ASD and also no such relationship in the NT group.

Furthermore, if individuals with ASD gaze away from the eyes because the mouth is more informative, one would expect longer fixation times on the mouth to have a beneficial effect on emotion recognition performance. In contrast, we found the reverse to be true: the greater the eye preference index (i.e., the fewer fixations on the mouth), the more correct emotions were classified by the autistic individuals. Moreover, emotions differ distinctively with respect to their diagnostic features across the face (Smith et al., 2005). For fearful and neutral faces, the eye region may contain the most important information about the emotion, whereas for happy faces, the mouth might be more distinctive. Although the number of fixation changes was modulated by the emotional expression for the NT group, which replicates previous findings (Gamer and Büchel, 2009), the ASD group showed no such effect. Also, if the interest in the mouth was greater (than in the eyes), eye movements away from the mouth might occur less often and would be slower for certain emotions, where the mouth is most informative, such as for happy faces. However, the ASD group gazed away from the mouth faster for happy faces than for fearful faces, albeit only on a descriptive level. Thus, the lack of benefit from fixations toward the mouth, together with the missing modulatory effect of facial emotion on gaze can be interpreted as further support of the hypothesis of active avoidance of the eyes in ASD.

It has been suggested that avoidance of the eyes may be mediated by an increased emotional arousal (Kylliainen and Hietanen, 2006). Thus, it is important to mention that even typically developed participants seem to experience facial expressions as more intense when they focus on the eye region (Gamer and Büchel, 2009). With respect to the mechanisms of typically developed gaze behavior; however, the reported increase in intensity has been suggested to underscore the saliency of the eye region. The saliency, in turn, leads to an increased orientation toward this important region of the face (when starting to gaze on another prominent feature, such as the mouth). The increase in experienced intensity as a response to direct eye contact thus represents a rather adaptive arousal in neurotypical controls, enhancing social cognitive functioning. The results of the current study show that direct eye contact leads to differential reflexive gaze behavior within the framework of atypical gaze in ASD: instead of persisting gaze at the eyes (to collect important information about the emotional state of others), individuals with ASD gazed more frequently away from the eyes, independent of the displayed emotional expression. In line with previous findings (see above), these gaze patterns could be interpreted as maladaptive consequences of arousal, oppositional to the arousal effect on controls. Thus, direct eye contact has a rather positive effect on social cognition in controls, whereas this effect is reversed for individuals with ASD. The opposite directional group effects in overall 
eye preference underline this hypothesis: in the present study, ASD specific gaze was associated with performance and social symptomatology but not with impairments in repetitive behavior and communication. Thus, these relationships further illustrate how the combination of avoidance and orientation reflect specific socioemotional dysfunctioning within the heterogeneous pathophysiology of ASD, rather than general illness severity.

In sum, we used a novel task to further disentangle avoidance and orientation-related reflexive gaze behavior. We found that atypical gaze in ASD was prominently influenced by an increased number of reflexive eye movements away compared with eye movements toward the eye region in ASD. Within the context of previous literature (Hutt and Ounsted, 1966; Richer and Coss, 1976; Dalton et al., 2005; Kylliainen and Hietanen, 2006; Joseph et al., 2008), more and at the same time faster eye movements away from the eyes compared with away from the mouth in ASD favor the assumption of a pronounced avoidance of eye contact compared with reduced orientation. We acknowledge that future studies shall apply variations in task design such as using inverted faces, directing initial fixations to the nose, or showing only specific facial features instead of the whole face. Moreover, subtle latency characteristics have to be further investigated with higher temporal resolution of eye movement recordings. Possible limitations of the current study arise from repeated reports of basic visual-processing differences in autism in the literature (Minshew et al., 1999; Goldberg et al., 2002; Luna et al., 2007; Thakkar et al., 2008). We did not find such general group differences, suggesting that the observed gaze patterns in ASD represent socioemotional dysfunctioning in ASD, rather than global differences in oculomotor functioning. There is, however, a clear need for further investigations in particular in relation to brain abnormalities (for cerebellar pathology, see Nowinski et al., 2005).

The results of the current study complement research on the role of the amygdala in autism (Baron-Cohen et al., 2000). In typically developed controls, the amygdala seems to be not only involved in orienting toward socially salient stimuli (Gamer and Büchel, 2009; Gamer et al., 2010), but also in fear and aversion processing (LeDoux et al., 1988; Adolphs et al., 1998, 2005; Phelps et al., 2004; Pessoa et al., 2005a,b; Seymour et al., 2005; Anders et al., 2008; Asghar et al., 2008; Hadjikhani et al., 2008). A behavioral dissociation of aversion- and orientation-related gaze in ASD provides an opportunity to simultaneously identify and dissociate sociocognitive functions potentially subserved by different amygdalar nuclei (Ball et al., 2007; Hurlemann et al., 2008; Gamer et al., 2010). With respect to the goal to gain a comprehensive understanding of autism pathophysiology, the present results provide crucial information for future research investigating atypical gaze in relation to brain function. Finally, interventions and behavioral training studies may benefit from considering these results: although strategies motivating attention toward social salient cues seem generally justified, special attention should be directed to the reduction of the aversive effect of direct eye contact.

\section{References}

Adolphs R, Tranel D, Damasio AR (1998) The human amygdala in social judgment. Nature 393:470-474.

Adolphs R, Gosselin F, Buchanan TW, Tranel D, Schyns P, Damasio AR (2005) A mechanism for impaired fear recognition after amygdala damage. Nature 433:68-72.

American Psychiatric Association (1994) Diagnostic and statistical manual of mental disorders, Ed 4. Washington, DC: American Psychiatric Press.

Anders S, Eippert F, Weiskopf N, Veit R (2008) The human amygdala is sensitive to the valence of pictures and sounds irrespective of arousal: an fMRI study. Soc Cogn Affect Neurosci 3:233-243.

Asghar AU, Chiu YC, Hallam G, Liu S, Mole H, Wright H, Young AW (2008) An amygdala response to fearful faces with covered eyes. Neuropsychologia 46:2364-2370.

Ball T, Rahm B, Eickhoff SB, Schulze-Bonhage A, Speck O, Mutschler I (2007) Response properties of human amygdala subregions: evidence based on functional MRI combined with probabilistic anatomical maps. PLoS ONE 2:e307.

Baron-Cohen S, Ring HA, Bullmore ET, Wheelwright S, Ashwin C, Williams SC (2000) The amygdala theory of autism. Neurosci Biobehav Rev 24:355-364.

Baron-Cohen S, Wheelwright S, Hill J, Raste Y, Plumb I (2001a) The "Reading the Mind in the Eyes" Test revised version: a study with normal adults, and adults with Asperger syndrome or high-functioning autism. J Child Psychol Psychiatry 42:241-251.

Baron-Cohen S, Wheelwright S, Skinner R, Martin J, Clubley E (2001b) The autism-spectrum quotient (AQ): evidence from Asperger syndrome/ high-functioning autism, males and females, scientists and mathematicians. J Autism Dev Disord 31:5-17.

Bölte S, Poustka F (2001) Factor structure of the Autism Diagnostic Interview-Revised (ADI-R): a study of dimensional versus categorical classification of autistic disorders. Z Kinder Jugendpsychiatr Psychother 29:221-229.

Dalton KM, Nacewicz BM, Johnstone T, Schaefer HS, Gernsbacher MA, Goldsmith HH, Alexander AL, Davidson RJ (2005) Gaze fixation and the neural circuitry of face processing in autism. Nat Neurosci 8:519-526.

Gamer M, Büchel C (2009) Amygdala activation predicts gaze toward fearful eyes. J Neurosci 29:9123-9126.

Gamer M, Zurowski B, Büchel C (2010) Different amygdala subregions mediate valence-related and attentional effects of oxytocin in humans. Proc Natl Acad Sci U S A 107:9400-9405.

Gillberg C, Gillberg C, Råstam M, Wentz E (2001) The Asperger Syndrome (and High-Functioning Autism) Diagnostic Interview (ASDI): a preliminary study of a new structured clinical interview. Autism 5:57-66.

Goeleven E, De Raedt R, Leyman L, Verschuere B (2008) The Karolinska directed emotional faces: a validation study. Cogn Emotion 22:1094-1118.

Goldberg MC, Lasker AG, Zee DS, Garth E, Tien A, Landa RJ (2002) Deficits in the initiation of eye movements in the absence of a visual target in adolescents with high functioning autism. Neuropsychologia 40:2039-2049.

Hadjikhani N, Hoge R, Snyder J, de Gelder B (2008) Pointing with the eyes: the role of gaze in communicating danger. Brain Cogn 68:1-8.

Hurlemann R, Rehme AK, Diessel M, Kukolja J, Maier W, Walter H, Cohen MX (2008) Segregating intra-amygdalar responses to dynamic facial emotion with cytoarchitectonic maximum probability maps. J Neurosci Methods 172:13-20.

Hutt C, Ounsted C (1966) Biological Significance of Gaze Aversion with Particular Reference to Syndrome of Infantile Autism. Behavioral Science 11:346-356.

Joseph RM, Ehrman K, McNally R, Keehn B (2008) Affective response to eye contact and face recognition ability in children with ASD. J Int Neuropsychol Soc 14:947-955.

Klin A, Jones W, Schultz R, Volkmar F, Cohen D (2002) Visual fixation patterns during viewing of naturalistic social situations as predictors of social competence in individuals with autism. Arch Gen Psychiatry 59:809-816.

Kylliäinen A, Hietanen JK (2006) Skin conductance responses to another person's gaze in children with autism. J Autism Dev Disord 36:517-525.

LeDoux JE, Iwata J, Cicchetti P, Reis DJ (1988) Different projections of the central amygdaloid nucleus mediate autonomic and behavioral correlates of conditioned fear. J Neurosci 8:2517-2529.

Leekam SR, Hunnisett E, Moore C (1998) Targets and cues: gaze-following in children with autism. J Child Psychol Psychiatry 39:951-962.

Lehrl S, Triebig G, Fischer B (1995) Multiple choice vocabulary test MWT as a valid and short test to estimate premorbid intelligence. Acta Neurol Scand 91:335-345.

Lord C, Rutter M, Le Couteur A (1994) Autism diagnostic interview-revised: a revised version of a diagnostic interview for caregivers of individuals with possible pervasive developmental disorders. J Autism Dev Disord 24:659-685. 
Luna B, Doll SK, Hegedus SJ, Minshew NJ, Sweeney JA (2007) Maturation of executive function in autism. Biol Psychiatry 61:474-481.

Lundqvist D, Flykt A, Öhmann A (1998) The Karolinska directed emotional faces-KDEF, CD-ROM from Department of Clinical Neuroscience, Psychology section, Karoliska Institutet.

Minshew NJ, Luna B, Sweeney JA (1999) Oculomotor evidence for neocortical systems but not cerebellar dysfunction in autism. Neurology 52:917-922.

Morris JS, Frith CD, Perrett DI, Rowland D, Young AW, Calder AJ, Dolan RJ (1996) A differential neural response in the human amygdala to fearful and happy facial expressions. Nature 383:812-815.

Nation K, Penny S (2008) Sensitivity to eye gaze in autism: is it normal? Is it automatic? Is it social? Dev Psychopathol 20:79-97.

Neumann D, Spezio ML, Piven J, Adolphs R (2006) Looking you in the mouth: abnormal gaze in autism resulting from impaired top-down modulation of visual attention. Soc Cogn Affect Neurosci 1:194-202.

Nowinski CV, Minshew NJ, Luna B, Takarae Y, Sweeney JA (2005) Oculomotor studies of cerebellar function in autism. Psychiatry Res 137:11-19.

Pelphrey KA, Sasson NJ, Reznick JS, Paul G, Goldman BD, Piven J (2002) Visual scanning of faces in autism. J Autism Dev Disord 32:249-261.

Pessoa L, Japee S, Ungerleider LG (2005a) Visual awareness and the detection of fearful faces. Emotion 5:243-247.

Pessoa L, Padmala S, Morland T (2005b) Fate of unattended fearful faces in the amygdala is determined by both attentional resources and cognitive modulation. Neuroimage 28:249-255.
Phelps EA, Delgado MR, Nearing KI, LeDoux JE (2004) Extinction learning in humans: role of the amygdala and vmPFC. Neuron 43:897-905.

Richer JM, Coss RG (1976) Gaze aversion in autistic and normal children. Acta Psychiatr Scand 53:193-210.

Schultz RT (2005) Developmental deficits in social perception in autism: the role of the amygdala and fusiform face area. Int J Dev Neurosci 23:125-141.

Senju A, Johnson MH (2009) Atypical eye contact in autism: models, mechanisms and development. Neurosci Biobehav Rev 33:1204-1214.

Seymour B, O’Doherty JP, Koltzenburg M, Wiech K, Frackowiak R, Friston K, Dolan R (2005) Opponent appetitive-aversive neural processes underlie predictive learning of pain relief. Nat Neurosci 8:1234-1240.

Smith ML, Cottrell GW, Gosselin F, Schyns PG (2005) Transmitting and decoding facial expressions. Psychol Sci 16:184-189.

Spezio ML, Adolphs R, Hurley RS, Piven J (2007a) Analysis of face gaze in autism using "Bubbles." Neuropsychologia 45:144-151.

Spezio ML, Huang PY, Castelli F, Adolphs R (2007b) Amygdala damage impairs eye contact during conversations with real people. J Neurosci 27:3994-3997.

Thakkar KN, Polli FE, Joseph RM, Tuch DS, Hadjikhani N, Barton JJ, Manoach DS (2008) Response monitoring, repetitive behaviour and anterior cingulate abnormalities in autism spectrum disorders (ASD). Brain 131:2464-2478. 